\title{
Study to determine whether intraoperative frozen section biopsy improves surgical treatment of non-melanoma skin cancer
}

\author{
GIOVANNI NICOLETTI ${ }^{1}$, FEDERICA BRENTA ${ }^{1}$, ALBERTO MALOVINI ${ }^{2}$, GAETANO MUSUMARRA ${ }^{1}$, \\ SILVIA SCEVOLA ${ }^{1}$ and ANGELA FAGA ${ }^{1}$ \\ ${ }^{1}$ Plastic and Reconstructive Surgery Unit, and ${ }^{2}$ Department of Computer Engineering and Systems Science, \\ University of Pavia, Salvatore Maugeri Research and Care Institute, I-27100 Pavia, Italy
}

Received October 19, 2012; Accepted December 4, 2012

DOI: $10.3892 /$ mco.2012.51

\begin{abstract}
Skin cancers are the most common types of cancer and their incidence has shown an increase of $\sim 4$ to $8 \%$ per year over the last 40 years. The majority of skin cancers $(\sim 97 \%)$ are non-melanoma skin cancers, mainly represented by basal cell $(80 \%)$ and squamous cell carcinomas $(20 \%)$. The use of intra-operative frozen section remains controversial in the surgical treatment of non-melanoma skin cancer, being commonly considered an optional tool, the reliability and effectiveness of which remain questionable. A large retrospective study was conducted to examine 670 surgical excisions of non-melanoma skin cancers of the head and neck in 481 patients over a period of nine years, between May, 2002 and December, 2011, at the Plastic and Reconstructive Surgery Unit of the University of Pavia, Salvatore Maugeri Research and Care Institute, Pavia, Italy. Results demonstrated the paradoxical ineffectiveness of an intra-operative frozen section biopsy in pursuing higher rates of radical excision in non-melanoma skin cancers. Nevertheless, a more detailed analysis on the use of frozen sections focusing on the various anatomical sites of the body demonstrated a reverse trend in the eyelids and canthi, where a higher success rate $(87.50$ vs. $69.77 \%)$ in the surgical treatment of non-melanoma skin cancers was obtained with the use of an intra-operative frozen section biopsy. Results of the present study suggested that intra-operative frozen section biopsy be routinely used in the surgical treatment of nonmelanoma skin tumors involving the eyelids and canthi.
\end{abstract}

Correspondence to: Dr Giovanni Nicoletti, Plastic and Reconstructive Surgery Unit, University of Pavia, Salvatore Maugeri Research and Care Institute, Via Salvatore Maugeri 10, I-27100 Pavia, Italy

E-mail: giovanni.nicoletti@unipv.it; giovanni.nicoletti@fsm.it

Key words: non-melanoma skin cancer, frozen section biopsy, basal cell carcinoma, squamous cell carcinoma, resection margins, surgical excision

\section{Introduction}

Skin cancers are the most common types of cancer and their incidence has shown an increasing of $\sim 4$ to $8 \%$ per year over the last 40 years (1). The majority of skin cancers $(\sim 97 \%)$ are non-melanoma skin cancers, mainly represented by basal cell (80\%) and squamous cell carcinomas (20\%) (2).

Despite the low mortality rates and the rare occurrence of metastases, non-melanoma skin cancers may be locally invasive and exhibit relapse following treatment, with significant morbidity $(3,4)$.

Recurrence is better defined as a persistent disease and is mainly correlated with inadequate local treatment (5-7). The use of intra-operative frozen section remains controversial in the surgical treatment of non-melanoma skin cancer, being commonly considered an optional tool, the reliability and effectiveness of which remain questionable (8-14).

A large retrospective study was therefore conducted to examine 670 surgical excisions of non-melanoma skin cancers of the head and neck in 481 patients over a period of nine years, between May, 2002 and December, 2011, at the Plastic and Reconstructive Surgery Unit of the University of Pavia, Salvatore Maugeri Research and Care Institute, Pavia, Italy. Additionally, indications, limits and effectiveness of the intraoperative frozen section biopsy are discussed in detail.

\section{Patients and methods}

Patient specimens. The samples included primitive, persistent and recurrent non-melanoma skin cancers of any size and in any site of the head and neck. The data source was the Salvatore Maugeri Research and Care Institute database of the University of Pavia (Pavia, Italy).

Patients. In total, 481 patients [212 females and 269 males, with a mean age of 69.4 years and a median of 71 (minimum 30 and maximum 97 years)] were enrolled in this study. A total of 670 surgical excisions of non-melanoma skin cancer were considered, with 588 lesions $(87.76 \%)$ being basal cell carcinomas and $82(12.24 \%)$ being squamous cell carcinomas. Intra-operative frozen section biopsy was required in 71 cases. Surgical excision was carried out with a $5-\mathrm{mm}$ margin in healthy tissue for clinically diagnosed basal cell carcinomas 
and a 1-cm margin for clinically diagnosed squamous cell carcinomas, in any site of the head and neck. The follow-up period ranged from a minimum of 1 year to a maximum of 9 years.

The present study was approved by the Ethics Committee of the Salvatore Maugeri Research and Care Institute on $16 / 05 / 2011$.

Frozen section technique. Each surgical biopsy undergoing an intra-operative frozen section examination was sent to the Anatomical Pathology laboratory as a fresh specimen. The surgeon marked $\geq 1$ spots on the specimen margins to be evaluated with a dye or a stitch, according to his/her own clinical assessment. A 3- to 4-mm section, including the marked spot, was cut from the specimen by the pathologist, mounted on a support using OCT glue (Diagnostic BioSystems, Inc., Pleasanton, CA, USA) and frozen at $-20^{\circ} \mathrm{C}$. Two 3- $\mu \mathrm{m}$ frozen sections, including the marked spot, were cut tangentially to the margin of the specimen, mounted on a slide and air-dried at room temperature. Sections were then stained using modified Giemsa Stain Solution (Merck KGaA, Darmstadt, Germany). The sections were then examined under light microscopy and the findings were verbally discussed with the surgeon by phone. In the majority of circumstances, two $3-\mu \mathrm{m}$ frozen sections sufficed for an intra-operative diagnosis. When discrepancies occurred, further sections were cut along the margin and prepared as above for microscopy examination. At the end of the procedure the specimens were defrosted and embedded in formalin for a standard histological examination.

Statistical analysis. Statistical analysis was carried out to assess any significant correlation between the use of frozen section biopsy and the success rate that was assumed to be the complete surgical excision.

The frozen section report was compared to the formalinembedded one in the same specimen to assess its classification accuracy. The rate of confirmed intra-operative diagnoses when compared with the corresponding formalin-embedded reports was estimated using the formula: Classification accuracy $(\%)=($ confirmed reports/total reports $) \times 100$. The binomial test (exact test) was applied to evaluate whether the classification accuracy reached by the use of an intra-operative frozen section biopsy was significantly higher compared to the fraction of correctly classified biopsies that could be obtained by chance.

The impact of the use of an intra-operative frozen section biopsy on the probability of obtaining a radical excision was evaluated using the two-tailed Fisher's exact test within the whole sample and within specific subclasses of tumors and anatomical localizations.

The same analysis was carried out on a reduced subset of patients characterized by tumors localized on the eyelids to evaluate the impact of an intra-operative frozen section biopsy on the success rate (complete surgical excision) within such a critical anatomical site. The one-tailed Fisher's exact test was employed to achieve this.

$\mathrm{P}<0.05$ was considered to indicate a statistically significant difference. Statistical analyses were carried out using the R statistical software (www.r-project.org/).
Table I. Distribution of intra-operative frozen section biopsies per anatomical site.

Anatomical site

Frozen sections, n (\%)

\begin{tabular}{lc}
\hline Head and neck & $71(100.00)$ \\
Nose & $34(47.89)$ \\
Eyelids & $17(23.94)$ \\
Cheek, chin, forehead & $8(11.27)$ \\
Lips & $7(9.86)$ \\
Ear & $3(4.22)$ \\
Scalp, neck & $2(2.82)$
\end{tabular}

\section{Results}

In the whole sample, of 670 non-melanoma skin cancers 597 demonstrated a complete surgical excision in healthy tissue $(89.10 \%)$. Within these completely excised tumors, recurrence was observed only in one case of basal cell carcinoma $(0.17 \%)$, one year post-operatively. Intra-operative frozen section biopsy was required in 71 of 670 cases. The distribution of the intra-operative frozen section biopsies per anatomical site is shown in Table I.

Following the histological examination, of the 71 frozen section biopsies $65(91.55 \%)$ were confirmed as intra-operative and $6(8.45 \%)$ were not (Table II). The success rate (complete surgical excision) in the intra-operative frozen section biopsy cohort was $84.51 \%$, with a completely excised tumor in 60 of 71 cases. The success rate in the resected cases without an intra-operative frozen section biopsy was $89.65 \%$, with a complete excision in 537 of 599 cases. The success rate per anatomical site, with and without the use of an intra-operative frozen section biopsy, is evident in Tables III and IV.

\section{Discussion}

Findings of the present study have demonstrated the paradoxical ineffectiveness of an intra-operative frozen section biopsy in pursuing higher rates of radical excision in nonmelanoma skin cancers. Such an outcome requires a more accurate interpretation with regard to the intrinsic limits of such a procedure. An intra-operative frozen section is potentially unreliable for two main reasons: intrinsic technical limits and subjective indication. Technically, a frozen specimen does not exhibit as many histological fine details as a formalin-embedded one. Therefore, the diagnostic accuracy of a frozen section report is always somewhat rough and imprecise. A frozen section is always required for one or more specimen margins, according to the surgeon's subjective clinical judgement to avoid potential failure of the surgeon and consequently the whole procedure.

Identifying a definite clinical margin of a non-melanoma skin carcinoma may be difficult in various circumstances. The sclerosing basal cell carcinoma usually features ill-defined borders resembling small patches of scleroderma with peripheral growth and central sclerosis. A multi-focal skin cancer within the context of a field cancerisation also shows 
Table II. Intra-operative frozen section biopsy report classification accuracy.

\begin{tabular}{|c|c|c|c|c|}
\hline \multirow[b]{2}{*}{ Anatomical site } & \multicolumn{2}{|c|}{ Intra-operative } & \multirow[b]{2}{*}{ Percentage } & \multirow[b]{2}{*}{ P-value } \\
\hline & $\begin{array}{l}\text { Frozen section } \\
\text { reports }\end{array}$ & $\begin{array}{l}\text { Confirmed } \\
\text { reports }\end{array}$ & & \\
\hline Overall & 71 & 65 & 91.55 & 0.062 \\
\hline Lips & 7 & 7 & 100.00 & 0.340 \\
\hline Eyelids & 17 & 16 & 94.12 & 0.171 \\
\hline Nose & 34 & 30 & 88.24 & 0.259 \\
\hline Cheek, chin, forehead & 8 & 7 & 87.50 & 0.736 \\
\hline Ear & 3 & 3 & 100.00 & 1 \\
\hline Scalp, neck & 2 & 2 & 100.00 & 1 \\
\hline
\end{tabular}

$\mathrm{P}<0.05$, statistically significant difference.

Table III. Radical excision rate per anatomical site, with and without intra-operative frozen section.

\begin{tabular}{|c|c|c|c|c|c|c|}
\hline \multirow[b]{2}{*}{ Tumor } & \multirow[b]{2}{*}{ Anatomical site } & \multirow[b]{2}{*}{$\mathrm{n}$} & \multicolumn{2}{|c|}{ Frozen section } & \multirow[b]{2}{*}{ OR $(95 \% \mathrm{CI})$} & \multirow[b]{2}{*}{ P-value } \\
\hline & & & Yes, n (\%) & No, n $(\%)$ & & \\
\hline \multirow[t]{7}{*}{ All } & Overall & 670 & $60(84.51)$ & $537(89.65)$ & $0.63(0.31-1.4)$ & 0.224 \\
\hline & Lips & 46 & $6(85.71)$ & $38(97.44)$ & $0.17(0-14.44)$ & 0.284 \\
\hline & Eyelids & 96 & $14(82.35)$ & $65(82.28)$ & $1.01(0.23-6.17)$ & 1.000 \\
\hline & Nose & 173 & $28(82.35)$ & $121(87.05)$ & $0.70(0.24-2.34)$ & 0.579 \\
\hline & Cheek, chin, forehead & 247 & $7(87.50)$ & $217(90.79)$ & $0.71(0.08-33.42)$ & 0.548 \\
\hline & Ear & 45 & $3(100.00)$ & $39(92.86)$ & - & 1.000 \\
\hline & Scalp, neck & 63 & $2(100.00)$ & $57(93.44)$ & - & 1.000 \\
\hline \multirow[t]{7}{*}{$\mathrm{BCC}$} & Overall & 588 & $52(82.54)$ & $474(90.29)$ & $0.51(0.24-1.15)$ & 0.079 \\
\hline & Lips & 38 & $5(83.33)$ & $31(96.88)$ & $0.17(0-15.17)$ & 0.294 \\
\hline & Eyelids & 91 & $12(80.00)$ & $62(81.58)$ & $0.90(0.20-5.65)$ & 1.000 \\
\hline & Nose & 161 & $25(80.65)$ & $114(87.69)$ & $0.59(0.19-2.02)$ & 0.381 \\
\hline & Cheek, chin, forehead & 213 & $5(83.33)$ & $191(92.27)$ & $0.42(0.04-21.03)$ & 0.397 \\
\hline & Ear & 30 & $3(100.00)$ & $26(96.30)$ & - & 1.000 \\
\hline & Scalp, neck & 55 & $2(100.00)$ & $50(94.34)$ & - & 1.000 \\
\hline \multirow[t]{7}{*}{$\mathrm{SCC}$} & Head & 82 & $8(100.00)$ & $63(85.14)$ & $\operatorname{Inf}(0.25$-Inf $)$ & 0.590 \\
\hline & Lips & 8 & $1(100.00)$ & $7(100.00)$ & - & - \\
\hline & Eyelids & 5 & $2(100.00)$ & $3(100.00)$ & - & - \\
\hline & Nose & 12 & $3(100.00)$ & $7(77.78)$ & $\operatorname{Inf}(0.06-\operatorname{Inf})$ & 1.000 \\
\hline & Cheek, chin, forehead & 34 & $2(100.00)$ & $26(81.25)$ & $\operatorname{Inf}(0.04-\operatorname{Inf})$ & 1.000 \\
\hline & Ear & 15 & $0(0)$ & $13(86.67)$ & - & - \\
\hline & Scalp, neck & 8 & $0(0)$ & $7(87.50)$ & - & - \\
\hline
\end{tabular}

$\mathrm{N}$, number of excisions; OR, odds ratio; CI, confidence interval; BCC, basal cell carcinoma; SCC, squamous cell carcinoma; Inf, the value cannot be estimated; -, the value cannot be estimated. $\mathrm{P}<0.05$, statistically significant difference.

ill-defined margins. Recurrence of a skin carcinoma following a failed previous surgical or conservative treatment therefore also poses the same issue.

In our experience, an intra-operative frozen section biopsy was required when the surgeon doubted the appropriateness of a previously outlined resection margin in an apparently healthy tissue. Such a subjectively based practice $a$ priori vitiated the sample distribution and randomization with distortion of the inclusion criteria and subsequent reduction of the cases with an intra-operative frozen section biopsy, compared to those without. This finding therefore explains the evident and statistically non-significant results of the comparison between the two groups, as well as the poor performance with the use of a supposedly advantageous tool. 
Table IV. Radical excision rate in the eyelids, with and without intra-operative frozen section.

\begin{tabular}{|c|c|c|c|c|c|}
\hline \multirow[b]{2}{*}{ Anatomical site } & \multirow[b]{2}{*}{$\mathrm{n}$} & \multicolumn{2}{|c|}{ Frozen section } & \multirow[b]{2}{*}{ OR (95\% CI) } & \multirow[b]{2}{*}{ P-value } \\
\hline & & Yes, n (\%) & No, n $(\%)$ & & \\
\hline Eyelids & 59 & $14(87.50)$ & $30(69.77)$ & 2.98 (0.68-Inf) & 0.145 \\
\hline Upper eyelid & 6 & $2(100.00)$ & $4(100.00)$ & - & - \\
\hline Lower eyelid & 45 & $9(90.00)$ & $26(74.29)$ & 3.05 (0.42-Inf) & 0.279 \\
\hline Medial canthus & 25 & $8(88.89)$ & $10(62.50)$ & 4.54 (0.54-Inf) & 0.174 \\
\hline Lateral canthus & 1 & $0(0)$ & $0(0)$ & - & - \\
\hline Lid margin & 8 & $4(80.00)$ & $2(66.67)$ & 1.83 (0.03-Inf) & 0.643 \\
\hline Naso-ocular angle & 32 & $10(90.91)$ & $14(66.67)$ & 4.79 (0.62-Inf) & 0.141 \\
\hline Lacrimal duct & 4 & $3(75.00)$ & $0(0)$ & - & - \\
\hline
\end{tabular}

$\mathrm{N}$, number of excisions; OR, odds ratio; CI, confidence interval; Inf, the value cannot be estimated; -, the value cannot be estimated. $\mathrm{P}<0.05$, statistically significant difference.

Despite such a relevant methodological defect that is correlated with the limits of a retrospective study to a certain extent, a noteworthy similarity in the success rate $(82.35$ vs. $82.28 \%$, odds ratio $>1$ ) between resected cases of intra-operative frozen section biopsy and those without was identified in tumors of the orbito-palpebral anatomical subgroup (96 records) (Table III). Thus, the analysis was refined within this subgroup by restricting the sample to an overall of 59 cases where the tumor involved the lid margin and the medial and lateral canthi. Tumors exhibit an aggressive attitude with potential early invasion of the orbit and skull base. A radical excision at these sites usually requires a technically demanding functional reconstruction (15). The refined statistical analysis is likely to eventually suggest the effectiveness of an intraoperative frozen section in obtaining a higher success rate ( 87.50 vs. $69.77 \%$, odds ratio 2.98 ) in the surgical treatment of non-melanoma skin cancers of the eyelids (Table IV).

This retrospective study provides a large and homogeneous sample of non-melanoma skin cancer in a western post-industrial society Caucasian population living in a temperate geographical area.

Treatment options available include curettage, desiccation, cryotherapy, topical regimens of 5 -fluorouracil (5-FU) or imiquimod (INN), photo-dynamic therapy and radiotherapy. However, surgery remains the mainstay in the treatment of non-melanoma skin cancer $(16,17)$. Radical excision of the neoplasm with a wide margin in healthy tissue is the key for success in this field (18). Accuracy in pre- and/or intraoperative outlining of the appropriate excision margin in healthy tissue remains an open issue. Several options and a discussion of their benefits and drawbacks have addressed the issue. Mohs micrographic surgery provides conservative excision of tissue with microscopic intra-operative examination of the excised tissue with proper orientation and colour coding of the specimens. The advisability of Mohs micrographic surgery in the treatment of non-melanoma skin cancer has long been debated in the literature, with equally motivated irreconcilable opinions from advocates and detractors (19). The main advantage of such an approach is the one-stage tumor excision in clear margins, while the disadvantages are its being time-consuming and patient discomfort. A previous study (20) using a large patient cohort investigated the long-term outcome of Mohs micrographic surgery for basal cell carcinomas of the face. Results of that study demonstrated a 5 -year recurrence rate of $4.5 \%$ for the lesions, $3.2 \%$ for primary tumors and $6.7 \%$ for recurrent ones. The rapid paraffin sections are a potential alternative to Mohs micrographic surgery as results obtained can be reported in $24 \mathrm{~h}$. Nevertheless, such a procedure is multi-staged, time-consuming and does not prevent patient discomfort. The frozen section is an optional tool utilized to intra-operatively assess one or more margins of a surgically excised specimen. Its advisability, reliability and effectiveness are as debated as Mohs micrographic surgery with a corresponding equal distribution of supporters and detractors (8-14).

In our experience, an intra-operative frozen section was required to check a complete excision of the neoplasm in one or more specimen marginal spots. In particular, an intraoperative frozen section was mostly required in the critical anatomical sites of the head, such as the nose, cheeks, eyelids, chin, lips and forehead. Thus, a potentially persistent disease from an incomplete primitive excision would demand a far more aggressive and disabling secondary surgery.

Our findings suggest the routine use of an intra-operative frozen section biopsy in the surgical treatment of non-melanoma skin tumors involving the eyelids and canthi.

Nevertheless, the effectiveness of an intra-operative frozen section biopsy is always correlated with the surgeon's subjective clinical assessment, even in the modern era of sophisticated and accurate objective diagnostic tools. The forthcoming use of the promising confocal laser scanning microscopy is likely to provide a non-invasive view to pre-operatively identify adequate resection margins $(21,22)$.

\section{Acknowledgements}

The authors wish to thank Gian Mario Pelizzoli for his much appreciated technical support. 


\section{References}

1. Housman TS, Feldman SR, Williford PM, et al: Skin cancer is among the most costly of all cancers to treat for the Medicare population. J Am Acad Dermatol 48: 425-429, 2003.

2. Chinem VP and Miot HA: Epidemiology of basal cell carcinoma An Bras Dermatol 86: 292-305, 2011.

3. Kumar V, Abba AK, Fausto N (eds.): Pathologic basis of disease. 7 th edition. Elsevier, India, 2004.

4. Kyrgidis A, Vahtsevanos K, Tzellos TG, et al: Clinical, histological and demographic predictors for recurrence and second primary tumors of head and neck basal cell carcinoma. A 1,062 patient-cohort study from a tertiary cancer referral hospital. Eur J Dermatol 20: 276-282,2010.

5. Friedman HL, Williams T, Zamora $\mathrm{S}$ and al-Assaad ZA: Recurrent basal cell carcinoma in margin-positive tumors. Ann Plast Surg 38: 232-235, 1997.

6. Goh BK, Ang P, Wu YJ, et al: Characteristics of basal cell carcinoma amongst Asians in Singapore and a comparison between completely and incompletely excised tumors. Int J Dermatol 45: 561-564, 2006.

7. Gulleth Y, Goldberg N, Silverman RP and Gastman BR: What is the best surgical margin for a basal cell carcinoma: a metaanalysis of the literature. Plast Reconstr Surg 126: 1222-1231, 2010 .

8. Manstein ME, Manstein CH and Smith R: How accurate is frozen section for skin cancers? Ann Plast Surg 50: 607-609, 2003.

9. Pluot M and Ducasse A: Pathology in the diagnosis and treatment of palpebral tumors. J Fr Ophtalmol 26: 77-83, 2003 (In French).

10. Cook BE Jr and Bartley GB: Treatment options and future prospects for the management of eyelid malignancies: an evidence-based update. Ophthalmology 108: 2088-2098, 2001.

11. van der Meer GT, Willemse F and Marck KW: Low 5-year recurrence rate after surgical excision of 126 basal cell carcinomas with frozen section analysis upon indication. Ned Tijdschr Geneeskd 145: 1409-1413, 2001 (In Dutch).
12. Ghauri RR, Gunter AA and Weber RA: Frozen section analysis in the management of skin cancers. Ann Plast Surg 43: 156-160, 1999.

13. Cataldo PA, Stoddard PB and Reed WP: Use of frozen section analysis in the treatment of basal cell carcinoma. Am J Surg 159: 561-563, 1990

14. Gloster HM and Brodland DG: The epidemiology of skin cancer. Dermatol Surg 22: 217-226, 1996.

15. Kumar P, Orton CI, McWilliam LJ and Watson S: Incidence of incomplete excision in surgically treated basal cell carcinoma: a retrospective clinical audit. Br J Plast Surg 53: 563-566, 2000.

16. Walker P and Hill D: Surgical treatment of basal cell carcinomas using standard postoperative histological assessment. Australas J Dermatol 47: 1-12, 2006.

17. Marchac D, Papadopoulos O and Duport G: Curative and aesthetic results of surgical treatment of 138 basal-cell carcinomas. J Dermatol Surg Oncol 8: 379-387, 1982.

18. Silverman MK, Kopf AW, Bart RS, et al: Reccurence rates of treated basal cell carcinomas. Part 3: surgical excision. J Dermatol Surg Oncol 18: 471-476, 1992.

19. Bialy TL, Whalen J, Veledar E, et al: Mohs micrographic surgery vs traditional surgical excision: a cost comparison analysis. Arch Dermatol 140: 736-742, 2004.

20. Smeets NW, Kuijpers DI, Nelemans P, et al: Mohs micrographic surgery for treatment of basal cell carcinoma of the face - results of a retrospective study and review of the literature. Br J Dermatol 151: 141-147, 2004.

21. Nori S, Rius-Díaz F, Cuevas J, et al: Sensitivity and specificity of reflectance-mode confocal microscopy for in vivo diagnosis of basal cell carcinoma: multicenter study. J Am Acad Dermatol 51: 923-930, 2004

22. Gerger A, Koller S, Weger W, et al: Sensitivity and specificity of confocal laser-scanning microscopy for in vivo diagnosis of malignant skin tumors. Cancer 107: 193-200, 2006. 